\title{
Efficient Deployment of UAV-powered Sensors for Optimal Coverage and Connectivity
}

\author{
Oktay Cetinkaya Geoff V. Merrett \\ Centre for IoT and Pervasive Systems \\ School of Electronics and Computer Science \\ University of Southampton, Southampton SO17 1BJ, UK \\ \{oc1y18,gvm\}@ecs.soton.ac.uk
}

\begin{abstract}
The Internet of Things (IoT) digitizes the physical world with wireless devices sensing their surroundings and delivering periodic notifications of parameters they are monitoring. However, this operation is bound by finite-capacity batteries, in which replenishment is practically infeasible due to the envisioned size of the IoT networks. By also considering the autonomous and self-sufficient service vision of the IoT paradigm, the need for novel approaches overcoming the energy constraints is evident. Here, unmanned aerial vehicles (UAVs) come into prominence. The UAVs can remotely energize wireless devices, via wireless power transfer (WPT), and thus guarantee reliable sensing coverage as well as longevity in the IoT domain. However, this can be only achieved by the precise alignment of both UAVs and wireless devices. Thus, this paper presents an efficient deployment strategy based on the circle packing problem, in which a lowerbound for the required number of wireless devices achieving optimal coverage is derived. The analysis, based on empirical measurements, reveals the design considerations for an energy harvesting (EH)-aided UAV scenario with regard to Federal Communications Commission (FCC) regulations, power consumption of wireless devices, and reporting frequency requirements of the IoT applications. Our results elaborate on a number of trade-offs, based on UAV, device, and medium characteristics, and provide realistic guidelines, achieving optimal coverage while meeting application requirements.
\end{abstract}

Index Terms-Wireless Power Transfer, Unmanned Aerial Vehicles, Internet of Things, Network Coverage, Deployment.

\section{INTRODUCTION}

The recent proliferation of unmanned aerial vehicles (UAVs) [1] draws considerable attention to the alleviation of energy constraints in the Internet of Things (IoT) [2]. The use of UAVs in (remote) powering of wireless devices, i.e. sensors, has a great potential to achieve perpetual and autonomous monitoring, especially for hard-to-reach mediums lacking in infrastructure and human support. The UAVs can enable rapid, on-demand, and high efficient power delivery thanks to the line-of-sight (LoS) air-to-ground links. This can greatly enhance service quality, i.e. reporting frequency or sensing coverage provided by energy-constrained wireless devices. The UAVs can also operate as (mobile) access points (APs), providing connectivity throughout the IoT networks. Furthermore, if the energy required for the UAV operation, including wireless power transfer (WPT) [3] to the devices, is provided by recharging stations that are capable of energy harvesting $(\mathrm{EH})[4]$, the vision of energy-neutral wirelesspowered networks [5] may become achievable.
The idea of WPT with EH-aided UAVs, however, is not entirely new [6]. There are also a plethora of studies focusing on different aspects of UAV operation to address the ongoing challenges [7], such as resource management, flight path and energy optimization, and interference minimization. Among them, efficient deployment of both UAVs and wireless devices come to the forefront [8]-[10], as it not only impacts the system performance but also intensifies other problems. However, the existing works on this topic partially or fully disregard some aspects that immensely affect the operation, which inevitably causes a discrepancy between the theory and practice. Thus, to tackle this problem and provide a more realistic analysis, this paper conducts an investigation on deployment/coverage by considering: 1) a limited source of power for WPT, i.e. an EH-aided UAV; 2) effective isotropic radiated power (EIRP) limitations enforced by the regulatory organizations, e.g. Federal Communications Commission (FCC); 3) power consumption of wireless devices; and 4) reporting frequency requirements of the IoT applications.

This work aims to convey a high-level discussion on the efficient deployment of network components, i.e. the UAV and wireless devices, to achieve optimum coverage over an event area of interest. With this agenda, we first derive the sensing range of wireless devices. Then, by using the circle packing problem (CPP) [11], we formulate a well-planned deployment strategy avoiding overlaps and oversteps, and thus enable efficient and interference-free operations. The analysis, based on empirical measurements, reveals the non-trivial relationships between the UAV attributes (e.g. altitude, directivity, energy budget, output power, transmission duration and frequency); device characteristics (e.g. quantity, sensing model, power consumption and conversion efficiency); medium specifications (e.g. path loss, event area); and application requirements (e.g. reporting frequency, throughput). In the end, this paper provides a lower-bound for the required number of wireless devices ensuring optimal coverage, which can be practically obtained by the careful selection of design parameters in a UAV-powered energy-neutral application scenario.

The remainder of this paper is organized as follows. We first introduce the system model in Sec. II, where the effect of directivity, adopted WPT model, rectenna operation, the FCC regulations, and the fundamental assumptions are explained. Accordingly, the (maximum) sensing range of wireless devices 
is derived. Sec. III outlines the conventional deployment models and formulates an efficient deployment strategy based on CPP, providing a lower-bound for the required number of wireless devices (by using the sensing range devised). This is followed by the numerical evaluation of the proposed model in Sec. IV to reveal the design guidelines needed to be followed to achieve optimal coverage while meeting application requirements. Finally, Sec. V concludes the paper.

\section{SySTEM MOdEL}

The network scenario shown in Fig. 1 is envisioned in a hierarchical order employing star topology between one UAV and $N$ wireless-powered devices ( $\mathrm{w}-\mathrm{p} D \mathrm{~s})$. The UAV retrieves energy from an EH recharging station, flies to the event area, and conveys the borrowed energy in a "point-to-multipoint" fashion to the identical $\mathrm{w}-\mathrm{p} D \mathrm{~s}$ deployed on the ground plane. During the WPT stage, the UAV do not move in the 3D space. The w-p $D$ s operate as battery-less sensors, i.e. they probe their vicinity for an application-defined parameter and notify higherlevel authorities via an IP-enabled remote AP, which can be the UAV in some cases. Since the $\mathrm{w}-\mathrm{p} D \mathrm{~s}$ are powered by the $\mathrm{UAV}$, they operate as long as they intercept enough power.

The UAV performs WPT with a directional antenna having a pencil-beam-like radiation pattern. For such an antenna, i.e. with one major lobe and very negligible minor lobs of the beam, the gain $G_{T}$ can be approximated by

$$
G_{T}=\left\{\begin{array}{crr}
\approx \frac{30000}{\theta_{B}^{2}}, & \frac{-\theta_{B}}{2} \leq \varphi \leq \frac{\theta_{B}}{2}, & \text { (major lobe) } \\
g(\varphi), & \text { otherwise, }, & \text { (minor lobes) }
\end{array}\right.
$$

where $\varphi$ is the sector angle, $\theta_{B}$ is the directional antenna halfpower beamwidth (HPBW) -both in degrees, $\approx 30000 / \theta_{B}^{2}$ is the maximum gain, and $g(\varphi)$ is the gain outside of the major lobe (including minor lobes), which can be neglected [12]. Note that (1) is for a symmetrical radiation pattern, in which the HPBWs in each plane are equal to each other, i.e. $\theta_{1 d}=\theta_{2 d}$.

For the scenario depicted in Fig. 1, the radiation of UAV resembles a cone with slant height $R_{R F}$, apex angle $\theta_{B}$, base radius $r_{u}$, and height $h$. Thus, from the projection of this radiation, the geographical (or event) area to be powered is $\Delta_{U A V}=\pi r_{u}^{2}=\pi R_{R F}^{2} \sin ^{2} \frac{\theta_{B}}{2}$. As seen, $\theta_{B}$ alters not only $G_{T}$ given by (1) but also $\Delta_{U A V}$. Any increment in $\Delta_{U A V}$, by keeping the other parameters constant, accordingly necessitates more than $N \mathrm{w}-\mathrm{p} D \mathrm{~s}$ to be deployed. However, the factor that affects $N$ the most is $R_{R F}$, which is the maximum distance that the UAV can deliver the minimum power required by the $\mathrm{w}-\mathrm{p} D \mathrm{~s}$ $\left(P_{R^{\text {min }}}\right)$. Let's consider this power as a function of Euclidean distance between the UAV and w-pDs. Then, $\left|P_{R^{\min }}(r)\right|$ for the diameter of the base circle will look like the probability density function of a normal distribution with zero mean and very high variance. This is the corollary of directivity, since $\theta_{B}$ is small; thus, $R_{R F} \approx h$. We, therefore, assume that the received power $P_{R}$ will be equal for each point in the base circle, i.e. the w-p $D$ s will receive the same $P_{R}$ irrespective of their location.

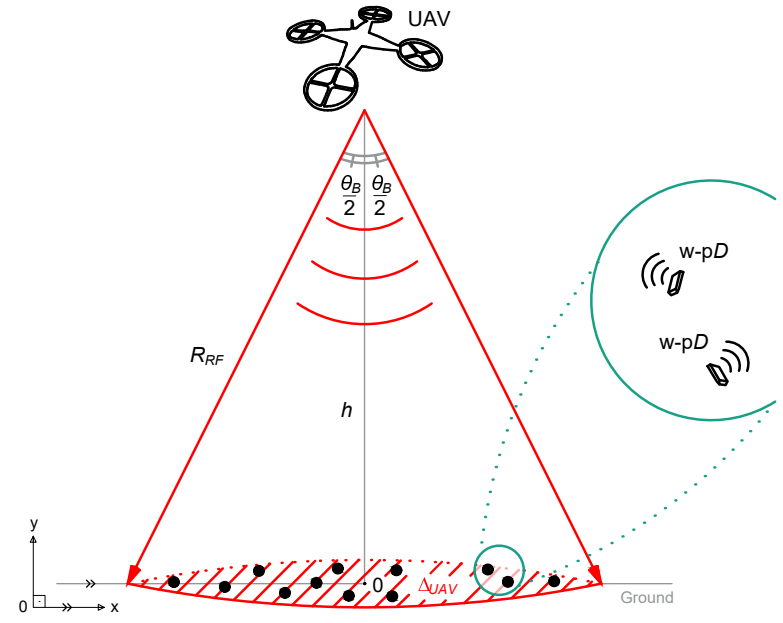

Figure 1: Orientation of network components (cross-sectional view).

Consequently, $P_{R}$ at distance $R_{R F}$ due to the UAV with transmit power $P_{T}$ can be given by Friis Transmission Equation [12] as

$$
P_{R}=P_{T} \frac{G_{T} G_{R} \lambda^{2}}{(4 \pi)^{2} R_{R F}^{v_{1}}}, \text { and } \lambda=\frac{c}{f},
$$

where $G_{R}$ is the antenna gain of the w-p $D \mathrm{~s}, \lambda$ is wavelength, $c$ is the speed of light, $f$ is the carrier frequency of RF waves transmitted by the UAV, and $v_{1}$ is the path loss exponent of the air-to-ground channel. This equation refers to the generic RF power transfer model, with no reflected signal between the $\mathrm{UAV}$ and $\mathrm{w}-\mathrm{p} D \mathrm{~s}$. It is also assumed that the $\mathrm{w}-\mathrm{p} D \mathrm{~s}$ are in $\operatorname{LoS}$ of the UAV, and there is no shadowing.

Contrary to expectations, the output power of the UAV in (2), cannot be altered casually; it is bound by the FCC regulations. FCC Part 15.247 rules declare that the maximum $P_{T}$ fed into the antenna cannot exceed $30 \mathrm{dBm}(1 \mathrm{~W})$ for the ISM bands, and the maximum effective isotropic radiated power (EIRP) is limited to $36 \mathrm{dBm}(4 \mathrm{~W})$ [13]. This indicates that increasing $G_{T}$ necessitates a proportional decrease in $P_{T}$, and vice versa, such that the total RF power radiated by the antenna remains the same, i.e. $4 \mathrm{~W}$ EIRP. For directional dispersion, on the contrary, there are some exceptions to the maximum EIRP. For example, in the $2.4 \mathrm{GHz}$ band, increasing $G_{T}$ to get an EIRP above $36 \mathrm{dBm}$ is allowed (up to $52 \mathrm{dBm}$ ), where $P_{T}$ must be reduced by $1 \mathrm{dBm}$ for every $3 \mathrm{dBi}$ increase of $G_{T}$. However, the physical size of the antennas increases with increasing $G_{T}$, which is impractical for sensors requiring small form factors. Besides, WPT at high frequencies is not useful as $P_{R}$ is inversely proportional to the square of the frequency. Thus, at $2.4 \mathrm{GHz}$, a power source increasing its EIRP (with $G_{T}$ ) has to decrease its $P_{T}$ to comply with the FCC regulations. As the UAV in our case has a fixed energy budget $E_{H}$, decreasing $P_{T}$ allows a longer duration of power transmission $t_{T}$, i.e. lengthened coverage lifetime. Although this sounds attractive, the duty cycle of the w-p $D$ s will accordingly be altered, which cannot be tolerated always due to the certain reporting frequency requirements of the IoT applications [14]. Furthermore, since decreasing $P_{T}$ (increasing $f$ ) will accordingly lower $P_{R}$, the w-p $D$ s may need to switch 
from power-neutral to energy-neutral operation [15], which is not desired for this particular scenario. Thus, these tradeoffs must be carefully considered during the system design to maximize the performance metric defined by the application.

It should also be noted that $P_{R}$ in (2) indicates the power intercepted by the w-p $D$ s (or the input power), which has to be converted into usable DC power, $P_{h a r}$, by a rectifying antenna (rectenna). Usually, a linear $\mathrm{EH}$ function is adopted for this operation, i.e. $P_{h a r}=\eta P_{R}$, where $\eta(0 \leq \eta<1)$ is the RFto-DC conversion efficiency of the rectenna. However, this model does not hold in practice due to the non-linearity of diodes, inductors, and capacitors used in the EH circuitry. Empirical results show that $\eta$ often increases with increasing $P_{R}$ and remains constant when $P_{R}$ exceeds the saturation power threshold, $P_{\text {sat }}$, of the harvester [3]. Thus, $\eta$ is neither a constant nor a linear parameter; it is a function of $P_{R}$, where $P_{R}$ is based on several variables as seen in (2). By also considering the sensitivity of rectenna, i.e. the minimum (threshold) power $P_{t h}$ required for its activation, the most accurate EH model can be given by the following piecewise linear function [16]

$$
P_{h a r}\left(P_{R}\right) \triangleq \begin{cases}0, & P_{R} \in\left[0, P_{t h}\right), \\ \eta\left(P_{R}\right) \cdot P_{R}, & P_{R} \in\left[P_{t h}, P_{\text {sat }}\right], \\ P_{\text {har }}\left(P_{\text {sat }}\right), & P_{R} \geq P_{\text {sat }},\end{cases}
$$

which is non-decreasing and continuous for all $P_{R} \in \mathbb{R}, P_{R} \geq 0$. By using curve-fitting tools for any empirical dataset, the function $\eta(\cdot)$ can be simply formulated as a polynomial, which gives a mathematically tractable expression with sufficient precision. In this work, the rectennas are assumed to operate in the ideal region, i.e. $P_{R} \in\left[P_{t h}, P_{s a t}\right]$ for all w-p $D$ s.

Now, let's suppose that the w-p $D$ s behave as omnidirectional radars, where they forward the power delivered by the UAV to their vicinity for the detection of any thing of interest at distance $r_{s}$. Since the $\mathrm{w}-\mathrm{p} D$ s are assumed to receive the same $P_{R}$ and are identical, they will radiate at the same transmit power $P_{T}^{\prime}$, which will result in equal sensing regions for all. Thus, the received, i.e. backscattered, signal strength at a w-p $D$, which is reflected from the target(s), can be characterized by the well-known radar equation [12] as

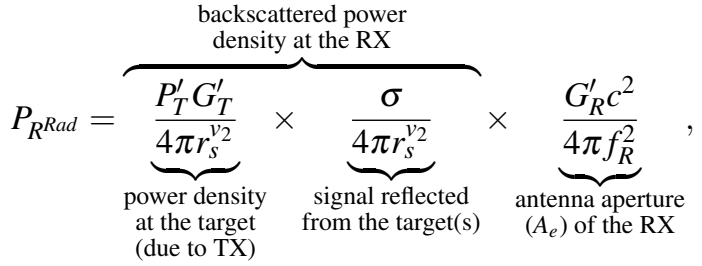

where $G_{T}^{\prime}$ and $G_{R}^{\prime}$ are respectively the transmitter (TX) and receiver $(\mathrm{RX})$ antenna gain of the $\mathrm{w}-\mathrm{p} D, \sigma$ is the radar crosssection of the target(s), and $v_{2}$ is the path loss exponent on the ground plane. Here, the w-p $D$ s will use the same antenna for TX and RX, i.e. monostatic radar, and thus $G_{T}^{\prime}=G_{R}^{\prime}=G_{R a d}$. Furthermore, due to losses and savings for future operations, e.g. processing of the sensed parameters and their transfer to the AP, the w-p $D$ s will only have a portion of $P_{\text {har }}$ to forward, i.e. $P_{T}^{\prime}=\beta P_{\text {har }}\left(P_{R}\right)=\beta \eta\left(P_{R}\right) P_{R}$, where $\beta$ is the loss factor.
In (4), if $r_{s}$ is assumed as the maximum distance that a w-p $D$ can reach, $P_{R_{\text {Rad }}}$ turns into the receiver sensitivity, i.e. the minimum power that has to be received to detect a target. For radars, $P_{R^{R a d}}^{\min }$ is given by $k T_{0} B F(S / N)_{\min }$, where $k$ is the Boltzmann constant, $T_{0}$ is the absolute temperature of the $\mathrm{w}-\mathrm{p} D$ input, $B$ is the $\mathrm{w}-\mathrm{p} D$ bandwidth, $F$ is noise factor, and $(S / N)_{\min }$ is the minimum signal-to-noise ratio needed to process a signal. The region to be probed by a w-p $D$ will therefore be a small circle with radius $r_{s} \max$, i.e. sensing range. By substituting (1), (2), and (3) in (4), and rearranging the resulting equation, $r_{s^{\max }}$ can be expressed as

$$
r_{S^{\max }}=\left(\frac{95.735 \times 10^{-3} \beta \eta\left(P_{R}\right) P_{T} G_{R} G_{R a d}^{2} \sigma c^{4}}{f^{2} f_{R}^{2} \theta_{B}^{2} R_{R F}^{v_{1}} k T_{0} B F(S / N)_{\text {min }}}\right)^{\frac{1}{2 v_{2}}} .
$$

It should be noted that sensing range for any sensor can be alternatively given by $r_{s}=\left(P_{T}^{\prime} / \mu\right)^{1 / \xi}$ [17], instead of the radar assumption, where $\mu$ is an application-dependent constant, and $\xi$ is a parameter referring to the power consumption model. In conclusion, (5) -or the above-given equation, can be used to designate relevant design parameters enabling effective sensing coverage in the given deployment scenario.

\section{W-PD DEPLOYMENT: OPtimal COVERAGE}

To ensure optimal coverage over the event area $\Delta_{U A V}$, the network must be carefully designed according to the UAV, $\mathrm{w}-\mathrm{p} D$, and medium characteristics. Here, the coverage refers to sensing quality of the network, and $k$-coverage implies that every single point in $\Delta_{U A V}$ is sensed by at least $k$ $(0 \leq k \leq N)$ w-p $D$ s. For coverage, two conventional models stand out: random, i.e. no strategy, and grid-type or wellplanned deployment, as shown in Fig. 2. Regardless of the adopted model, $P_{\text {har }}$ given by (3) must be $\geq P_{R^{\min }}$ of the w-pDs. This is to reliably sense $\Delta_{U A V}$ and deliver the measurements to a remote AP, i.e. guaranteed coverage over the event area and connectivity in the network.

The key factor deriving $k$-coverage for a random deployment is the sensing range $r_{s} \max$ of the $\mathrm{w}-\mathrm{p} D \mathrm{~s}$. This can be simply determined via stochastic geometry [18], where $k$ depends on the homogeneous Poisson point process of the $\mathrm{w}-\mathrm{p} D$ density.

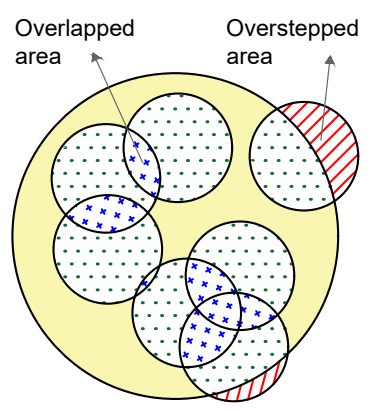

(a)

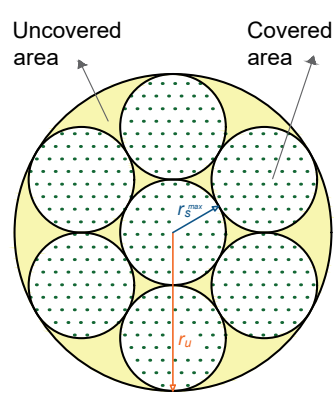

(b)
Figure 2: Possible deployment models: (a) no strategy (random); (b) well-planned (grid-type). Small circles represent the sensing regions of the w-pDs, where the big circle is $\Delta_{U A V}$. 
Stochastic geometry allows derivation of a tractable expression for $k$ ensuring certain coverage probability, assuming that $\mathrm{w}-\mathrm{p} D \mathrm{~s}$ are randomly and uniformly positioned over the event area. However, $k$, calculated by stochastic geometry is denoted as the worst-case scenario since the sensing regions of $\mathrm{w}-\mathrm{p} D \mathrm{~s}$ may overlap, which would necessitate more $\mathrm{w}-\mathrm{p} D \mathrm{~s}$ to be deployed for achieving the same coverage rate, $D_{v}$. By also considering that radar assumption may not properly work in the case of random positioning due to false alarms, gridtype deployment seems preferable as it may achieve a higher $D_{v}$ for the same $k$ or the same $D_{v}$ with less than $k$ w-pDs.

For a well-planned deployment, the $\mathrm{w}-\mathrm{p} D$ s must be positioned in a way such that each point in $\Delta_{U A V}$ falls into only one w-pD's sensing range, i.e. no overlaps occur (constraint \#1). In addition, for the most efficient utilization of the w-p $D$ s, the areas that they cover must stay within $\Delta_{U A V}$, i.e. no oversteps allowed (constraint \#2). These will potentially allow $N$ to be minimized at the cost of initial deployment. By considering a circular sensing region of $\pi r_{s}^{2}$ max for each $\mathrm{w}-\mathrm{p} D$, the problem can be formulated as

$$
\begin{array}{ll}
\max & D_{v}=\frac{N r_{s}^{2}{ }^{\max }}{R_{R F}^{2} \sin ^{2} \frac{\theta_{B}}{2}}, \\
\text { s.t. } & \left(x_{i}-x_{j}\right)^{2}+\left(y_{i}-y_{j}\right)^{2} \geq\left(2 r_{s} \max \right)^{2}, i \neq j \in\{1, . ., N\}, \\
& x_{i}^{2}+y_{i}^{2} \leq\left(R_{R F} \sin \frac{\theta_{B}}{2}-r_{s} \max \right)^{2}, x_{i}, y_{i} \in \mathbb{R}, i \in\{1, . ., N\}, \\
& R_{R F} \sin \frac{\theta_{B}}{2} \geq r_{s} \max \geq 0,
\end{array}
$$

where $x_{i}$ and $y_{i}$ refers to the Cartesian coordinates of $\mathrm{w}-\mathrm{p} D_{i}$ on the ground plane (considering $\Delta_{U A V}$ 's center as the origin), (7) is constraint \#1 and (8) is constraint \#2, explained above. Here, (7) guarantees also that there will be no collision or interference between the w-p $D$ s. In other terms, if the distance between the $\mathrm{w}-\mathrm{p} D$ and a target is $r_{s^{\max }}$, there cannot be any w-p $D$ closer than $r_{s}$ max to that particular target. Finally, (9) is an obvious upper bound for the sensing range.

Solving (6), i.e. maximizing $D_{v}$ with a minimum $N$, is not easy due to the above-formulated constraints, the FCC regulations, and a number of variables that depend on UAV attributes. Thus, we model (6) by using the circle packing problem (CPP) [11]. In CPP, $N$ circles, which refer to the number of $\mathrm{w}-\mathrm{p} D \mathrm{~s}$, and their corresponding (sensing) areas -for our case, are arranged in a given surface, i.e. $\Delta_{U A V}$, such that the packing density, $D_{v}$, is maximized without any overlaps and oversteps. It should be noted that CPP in a given surface is often intractable [8], i.e. it is not likely to constitute a general packing strategy that is optimal for any $N$. For example, both mirror and square symmetry achieve the same $D_{v}(0.5556)$ for $N=5$, while pentagonal packing outperforms those two with $D_{v}$ of 0.6852 . Depending on $N$, new symmetry types, which were not applicable earlier or performed worse, may stand out.

In (6), for a fixed $\Delta_{U A V}$, i.e. constant denominator, $r_{s^{m}}$ ax decreases for increasing $N . D_{v}$, on the other hand, is not strictly increasing, which makes the problem more difficult to solve. In CPP, $N$ is usually given and the main goal is to find the radius of small circles covering a bigger circle (or container) for the highest $D_{v}$. However, in our case, since the energy budget of the UAV is limited, we can calculate the maximum radius that the circles can have, i.e. $r_{s} \max$, which gives the minimum number of $N$ to be deployed. Thus, CPP will provide a lowerbound for $N$, which is again subject to several variables.

Now, let's define $\chi$ as the ratio of the UAV base radius to $\mathrm{w}-\mathrm{p} D$ sensing range, i.e. $\chi=\frac{R_{R F} \sin \left(\theta_{B} / 2\right)}{r_{\text {max }}}$, and assume $N$ is the required number of $\mathrm{w}-\mathrm{p} D$ s to pack the area covered by UAV. In [19], an upper bound for $\chi$ is given as

$$
\chi \leq \frac{\sqrt{\left(4 q_{h}-1\right)^{2}+16 q_{h}(N-1)}-1}{4 q_{h}}+1,
$$

where $q_{h}$ denotes the density of hexagonal lattice packing, i.e. $q_{h}=\frac{\pi}{2 \sqrt{3}}$. By using (10) and [20], $N$ can be conjectured as

$$
N \geq\left\lceil q_{h} \chi^{2}-\left(2 q_{h}-\frac{1}{2}\right) \chi+\zeta\right\rceil,\{\chi \in \mathbb{R}: \chi \geq 1(\text { from }(9))\},
$$

where $\zeta=2$ for $3 \leq \chi<10$, and $=1$ otherwise. By using $N$ from (11), the coverage rate can be calculated as $D_{v}=\frac{N}{\chi^{2}}$. It should be noted that the maximum $D_{v}$ that can be achieved by hexagonal lattice packing is $\approx 0.907$, i.e. $q_{h}$.

\section{Simulation Results And Discussion}

For simulations, we consider the UAV-based WPT at $4 \mathrm{~W}$ EIRP over $f$ of $868 \mathrm{MHz}$ (unless otherwise stated) in a suburban area, where the w-p $D$ s have $G_{R}=G_{\text {rad }}=6 \mathrm{dBi}$ and $P_{R^{\text {Rad }}}^{\text {min }}$ of $10^{-9}$. The other constant parameters are as follows: $c=3 \times 10^{8} \mathrm{~m} / \mathrm{s}, f_{R}=2.45 \mathrm{GHz}, v_{1}=2.2$ [21], $v_{2}=4.7$ [22], $\sigma=0.01 \mathrm{~m}^{2}$, and $\beta=0.8$. Furthermore, $\eta$ is calculated as a function of $P_{R}$ by using real data outsourced from [23] and [24]. Fig. 3(a) and (b) depict the fitted functions of the used rectenna designs operating at 0.868 and $2.45 \mathrm{GHz}$, respectively.

Fig. 4(a) and (b) show the number of w-pDs, $N$, and the coverage rate, $D_{v}$ (corresponds to each $N$ ) as a function of altitude, $h$. Not surprisingly, as the height of UAV increases, more $N$ is needed. This is because the w-pDs receive less $P_{R}$ and $\Delta_{U A V}$ gets larger with increasing $h$. For a fixed $h$, lower $G_{T}$ (or higher $\theta_{B}$ ) necessitates more $N$ to be deployed, since the power dispersion is performed towards a wider area, i.e. larger $\Delta_{U A V}$ to be packed. $D_{v}$, on the other hand, is not always increasing due to the constraints explained in the previous section. Fig. 4(c) illustrates the change in $N$ and $D_{v}$ as well as

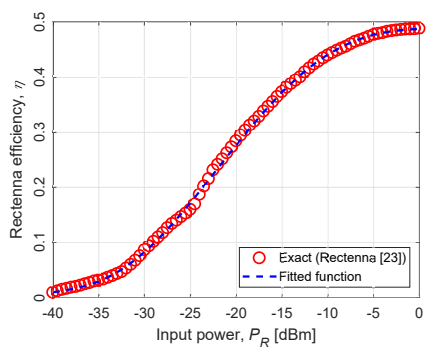

(a)

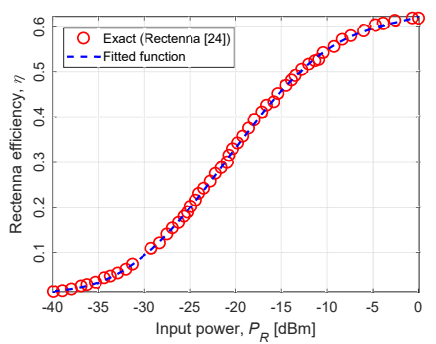

(b)
Figure 3: The rectenna efficiency, $\eta$, as a function of input power, $P_{R}$ : (a) for the rectenna proposed in [23] (@ $f_{\text {rect }}=868 \mathrm{MHz}$ ); (b) for the rectenna proposed in [24] (@ $\left.f_{\text {rect }}=2.45 \mathrm{GHz}\right)$. 


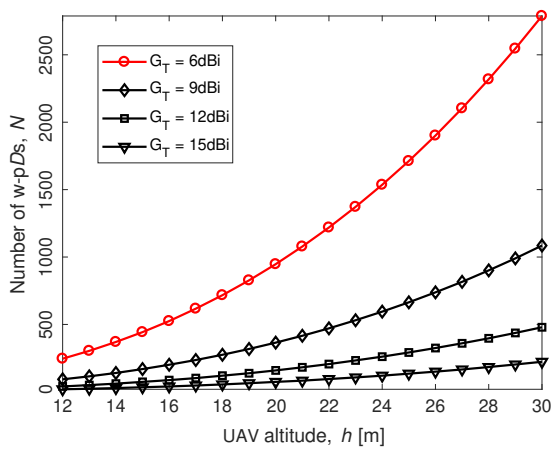

(a)

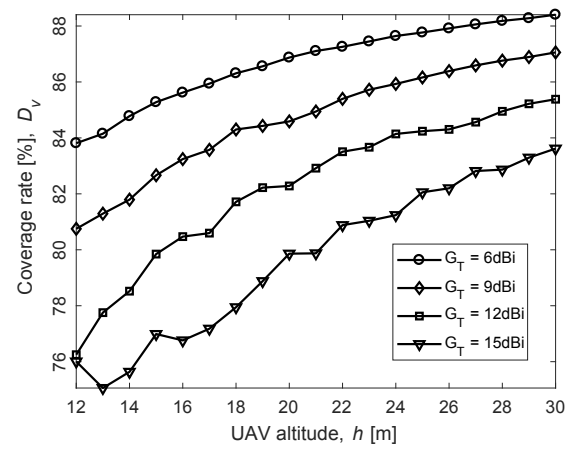

(b)

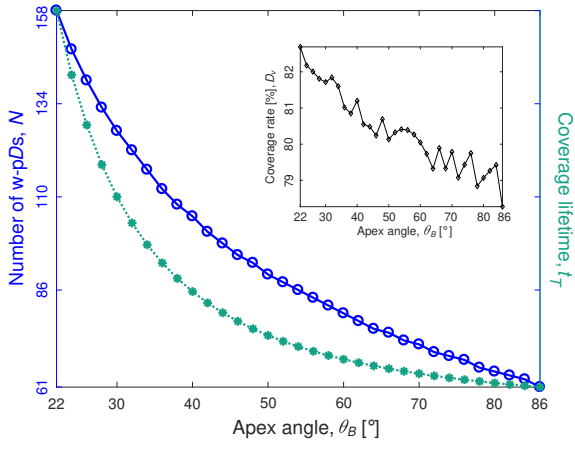

(c)

Figure 4: The number of w-p $D \mathrm{~s}, N$, coverage rate, $D_{v}$, and coverage lifetime, $t_{T}$, for UAV altitude, $h$, and apex angle, $\theta_{B}$ (4W EIRP @ $f=868 \mathrm{MHz}$ ): (a) $N$ vs. $h$; (b) $D_{v}$ vs. $h$ (for $G_{T}$ of $6,9,12$, and $15 \mathrm{dBi}$ ); (c) $N$ and $D_{v}$ vs. $\theta_{B}$ and $t_{T}$ vs. $\theta_{B}$ (for $\Delta_{U A V}$ of $140 \mathrm{~m}^{2}$ ).

coverage lifetime $t_{T}$ for varying $\theta_{B}$. Due to the fixed energy budget of the UAV, $E_{H}$, the duration of power transmission, $t_{T}$, is limited by $P_{T}$. In other terms, $t_{T}$ will decrease proportionally to any increase in $P_{T}$, and vice versa, as a corollary of $E_{H}=P_{T} \times t_{T}$, since the UAV is assumed to perform WPT always at the maximum output power allowed by the FCC (4W EIRP). Hence, as seen in Fig. 4(c), increasing $\theta_{B}$, i.e. decreasing $G_{T}$, will allow $P_{T}$ to be increased as much as possible without violating the maximum EIRP rule, which will accordingly shorten $t_{T}$. It should be noted that dynamic change in $\theta_{B}\left(\right.$ or $\left.G_{T}\right)$ is not possible in practice. This is just to provide more insights on the characteristics of directional WPT with a limited source of power.

Fig. 5 can be considered as a derivative of Fig. 4(a) and (b), where $N$ and $D_{v}$ are given as a function of $\Delta_{U A V}$. As seen, (unlike Fig. 4) $N$ is higher for higher $G_{T}$ at a fixed $\Delta_{U A V}$, since the UAV with higher $G_{T}$ has to climb up for keeping the $\Delta_{U A V}$ constant (due to the narrower $\theta_{B}$ ). In practice, some applications requiring coverage over a fixed event area or the medium of interest (e.g. urban areas) may have some restrictions on the lowest $h$, i.e. the closest altitude approachable. Thus, the UAV has to be equipped with the most suitable antenna to achieve the highest $D_{v}$ with a minimum $N$ while meeting the application requirements/complying with the specific regulations.

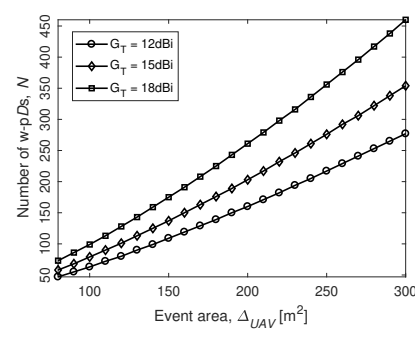

(a)

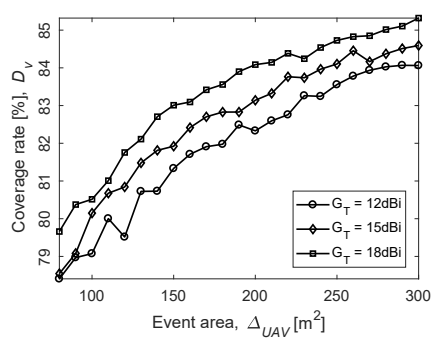

(b)
Figure 5: The number of w-p $D \mathrm{~s} N$ and coverage rate $D_{v}$ for the increasing size of event area $\Delta_{U A V}$ : (a) $N$ vs. $\Delta_{U A V}$; (b) $D_{v}$ vs. $\Delta_{U A V}$ (for $G_{T}$ of 12, 15, and $18 \mathrm{dBi}, 4 \mathrm{~W}$ EIRP @ $f=868 \mathrm{MHz}$ ).

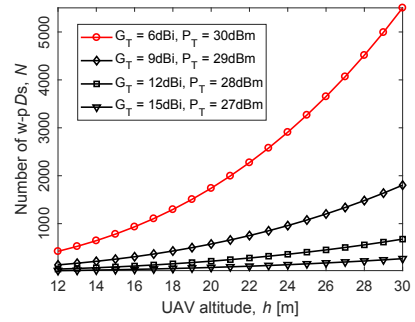

(a)

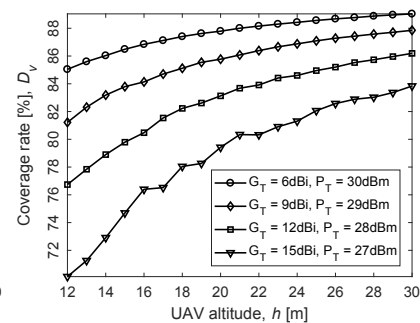

(b)
Figure 6: The effect of increasing EIRP on the number of w-p $D \mathrm{~s}, N$, and coverage rate, $D_{v}$, for varying UAV altitude, $h$ : (a) $N$ vs. $h$; (b) $D_{v}$ vs. $h$ (for EIRP of $\approx 4,6.31,10$, and $15.85 \mathrm{~W} @ f=2.45 \mathrm{GHz}$ ).

Fig. 6 compares the same parameters in Fig. 4(a) and (b) but for an increasing EIRP at $f$ of $2.45 \mathrm{GHz}$. Despite the increase in EIRP (from 4 to $15.85 \mathrm{~W}$ ), the required number of $\mathrm{w}-\mathrm{p} D \mathrm{~s}$, $N$, has considerably increased since the signal fading is severer at higher $f$, i.e. $P_{R} \propto 1 / f^{2}$. Thus, $P_{R}$ reduces by $\approx 8$ times (for the same EIRP) when $f$ changes from $868 \mathrm{MHz}$ to $2.45 \mathrm{GHz}$. Note that the red lines in Fig. 4(a) and Fig. 6(a) correspond to the same EIRP ( $4 \mathrm{~W}$ or $36 \mathrm{dbm}$, i.e. $6 \mathrm{dBi}$ of $G_{T}+30 \mathrm{dBm}$ of $P_{T}$ ). Even for the highest EIRP considered $(15.85 \mathrm{~W}), P_{R}$ is still half of the value that is achieved at $868 \mathrm{MHz}$ with $4 \mathrm{~W}$ EIRP. Reducing $P_{R}$ also reduces $\eta$, as can be observed from Fig. 3, which adversely affects $r_{s^{m}}$, and thus $N$. Hence, if the application necessitates the use of $2.45 \mathrm{GHz}$ band for the WPT, the UAV should operate on the highest EIRP (considering the antenna size) at the lowest altitude possible. In such a way, the effect of frequency and fading components can be minimized, achieving similar performance to that of the sub-GHz bands. Yet, the increase in the EIRP may affect the duty cycle or reporting frequency of the $\mathrm{w}-\mathrm{p} D \mathrm{~s}$ since the UAV will need frequent replenishment due to the increased power output, i.e. more trips to the recharging station (duration of which is orders of magnitude longer than $t_{T}$ ). Furthermore, in addition to the lower $P_{R}$ received by the w-p $D$ s, the throughput at the AP will decrease due to the total time elapsed. These trade-offs point out the need for a more inclusive and complex optimization problem, which is not in the scope of this paper. 


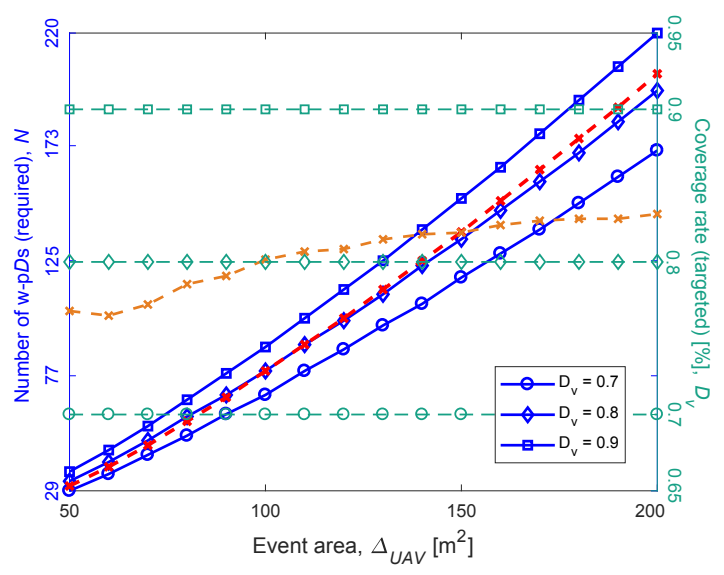

Figure 7: The number of $\mathrm{w}-\mathrm{p} D \mathrm{~s}$ required, $N$, to achieve different target coverage rates, $D_{v}$, when increasing size of event area $\Delta_{U A V}$ $\left(\theta_{B} \approx 30^{\circ}, 4 \mathrm{~W}\right.$ EIRP @ $\left.f=868 \mathrm{MHz}\right)$. The dashed red line shows $N$ that can be fit in the given $\Delta_{U A V}$ (with respect to system parameters), where achievable $D_{v}$ for that $N$ is shown in orange.

Fig. 7 shows the minimum number of $\mathrm{w}-\mathrm{p} D$ s required to satisfy a targeted coverage rate over the event area of interest. For any given $\Delta_{U A V}$ (from 50 to $200 \mathrm{~m}^{2}$ ), a $D_{v}$ of 0.7 can be achieved by a UAV having $G_{T}$ of $15 \mathrm{dBi}$ and transmitting $4 \mathrm{~W}$ of EIRP at $868 \mathrm{MHz}$. However, for $D_{v}=0.8, \Delta_{U A V}$ must be at least $100 \mathrm{~m}^{2}$. This is a corollary of CPP, i.e. the more w$\mathrm{p} D$ s to be deployed, the higher $D_{v}$ will be achieved (which occurs only if $\Delta_{U A V}$ gets larger or $r_{s} \max$ becomes shorter for the same $\left.\Delta_{U A V}\right)$. For the envisioned setting, 0.9 of $D_{v}$ is not achievable as it needs more than $N$ w-p $D$ s that can fit in the given $\Delta_{U A V}$. Depending on $\theta_{B}, P_{T}, f$, and $\Delta_{U A V}$, achievable $D_{v}$ varies with $N$. Thus, optimal designation of the relevant design parameters is of utmost importance to meet the requirements (e.g. minimum $N$, maximum $D_{v}$, certain reporting frequency, and highest throughput) set by the applications.

\section{CONCLUSIONS}

This paper investigates the optimal coverage in the IoT networks with a minimum number of $\mathrm{w}-\mathrm{p} D \mathrm{~s}$, which are remotely powered by an energy-limited UAV with a directional antenna. First, the maximum sensing range of the $\mathrm{w}-\mathrm{p} D \mathrm{~s}$ was derived as a function of UAV altitude, output power, and directivity. Then, by using the CPP theorem, an efficient deployment strategy avoiding the overlaps and oversteps, and thus providing a lower-bound for the required number of $\mathrm{w}-\mathrm{p} D \mathrm{~s}$ was proposed. The analysis revealed the design considerations for maximum coverage with regard to the FCC regulations, realistic rectenna operation, and minimum power and reporting frequency requirements of the w-p $D$ s. Future work will focus on the optimization of the afore-mentioned trade-offs to maximize the throughput in the UAV-powered energy-neutral networks.

\section{ACKNOWLEDGMENT}

This work was supported by the UK EPSRC under EP/P010164/1. Simulation data can be found at DOI: 10.5258/SOTON/D1221.

\section{REFERENCES}

[1] S. Hayat, E. Yanmaz, and R. Muzaffar, "Survey on Unmanned Aerial Vehicle Networks for Civil Applications: A Communications Viewpoint," IEEE Commun. Surv. Tutor, vol. 18, no. 4, pp. 2624-2661, FQ 2016.

[2] O. B. Akan et al., "Internet of Hybrid Energy Harvesting Things," IEEE Internet of Things Journal, vol. 5, no. 2, pp. 736-746, Apr. 2018.

[3] X. Lu et al., "Wireless Networks With RF Energy Harvesting: A Contemporary Survey," IEEE Communications Surveys Tutorials, vol. 17, no. 2, pp. 757-789, Secondquarter 2015.

[4] O. Cetinkaya and O. B. Akan, "Electric-Field Energy Harvesting in Wireless Networks," IEEE Wireless Communications, vol. 24, no. 2, pp. 34-41, Apr. 2017

[5] O. Cetinkaya et al., "Energy-Neutral Wireless-Powered Networks," IEEE Wireless Communications Letters, vol. 8, no. 5, pp. 1373-1376, Oct. 2019.

[6] T. Long et al., "Energy Neutral Internet of Drones," IEEE Communications Magazine, vol. 56, no. 1, pp. 22-28, Jan. 2018.

[7] M. Mozaffari et al., "A Tutorial on UAVs for Wireless Networks: Applications, Challenges, and Open Problems," IEEE Communications Surveys Tutorials, vol. 21, no. 3, pp. 2334-2360, thirdquarter 2019.

[8] — , "Efficient Deployment of Multiple Unmanned Aerial Vehicles for Optimal Wireless Coverage," IEEE Communications Letters, vol. 20, no. 8, pp. 1647-1650, Aug. 2016.

[9] M. Alzenad et al., "3-D Placement of an Unmanned Aerial Vehicle Base Station (UAV-BS) for Energy-Efficient Maximal Coverage," IEEE Wireless Communications Letters, vol. 6, no. 4, pp. 434-437, Aug. 2017.

[10] H. He et al., "Joint Altitude and Beamwidth Optimization for UAVEnabled Multiuser Communications," IEEE Communications Letters, vol. 22, no. 2, pp. 344-347, Feb. 2018.

[11] M. Hifi and R. M'Hallah, "A Literature Review on Circle and Sphere Packing Problems: Models and Methodologies," https://www.hindawi.com/journals/aor/2009/150624/abs/, 2009.

[12] C. A. Balanis, Antenna Theory: Analysis and Design. John Wiley \& Sons, Feb. 2016.

[13] "Federal Communications Commission CFR, Title 47, Volume 1, Part 15," https://www.govinfo.gov/app/details/CFR-2010-title47-vol1, 2010.

[14] O. Cetinkaya and O. B. Akan, "Electric-Field Energy Harvesting From Lighting Elements for Battery-Less Internet of Things," IEEE Access, vol. 5, pp. 7423-7434, 2017.

[15] S. T. Sliper et al., "Energy-driven computing," Philosophical Transactions of the Royal Society A: Mathematical, Physical and Engineering Sciences, vol. 378, no. 2164, p. 20190158, Feb. 2020.

[16] P. N. Alevizos and A. Bletsas, "Sensitive and Nonlinear Far-Field RF Energy Harvesting in Wireless Communications," IEEE Transactions on Wireless Communications, vol. 17, no. 6, pp. 3670-3685, Jun. 2018.

[17] M. Cardei et al., "Maximum network lifetime in wireless sensor networks with adjustable sensing ranges," in WiMob'2005), IEEE International Conference on Wireless And Mobile Computing, Networking And Communications, 2005., vol. 3, Aug. 2005, pp. 438-445 Vol. 3.

[18] B. Liu and D. Towsley, "A study of the coverage of large-scale sensor networks," in 2004 IEEE International Conference on Mobile Ad-Hoc and Sensor Systems (IEEE Cat. No.04EX975), Oct. 2004, pp. 475-483.

[19] D. W. Cantrell, "Packing unit circles in circles: New results," https://groups.google.com/d/msg/sci.math/SMr66MEH9k/PoMJIKhB3KwJ, Dec. 2008.

[20] E. Specht, "The best known packings of equal circles in a circle," http://hydra.nat.uni-magdeburg.de/packing/cci/cci.html\#Applications, Aug. 2014.

[21] E. Yanmaz, R. Kuschnig, and C. Bettstetter, "Channel measurements over 802.11a-based UAV-to-ground links," in 2011 IEEE GLOBECOM Workshops (GC Wkshps), Dec. 2011, pp. 1280-1284.

[22] D. Balachander, T. R. Rao, and G. Mahesh, "RF propagation investigations in agricultural fields and gardens for wireless sensor communications," in 2013 IEEE Conference on Information Communication Technologies, Apr. 2013, pp. 755-759.

[23] S. D. Assimonis, S.-N. Daskalakis, and A. Bletsas, "Sensitive and Efficient RF Harvesting Supply for Batteryless Backscatter Sensor Networks," IEEE Transactions on Microwave Theory and Techniques, vol. 64, no. 4, pp. 1327-1338, Apr. 2016.

[24] S.-E. Adami et al., "A Flexible 2.45-GHz Power Harvesting Wristband With Net System Output From -24.3 dBm of RF Power," IEEE Transactions on Microwave Theory and Techniques, vol. 66, no. 1, pp. 380-395, Jan. 2018 